\title{
Fluxograma utilizado para dor torácica em 523 pacientes admitidos na emergência do Hospital Unimed Recife III
}

\section{Flowchart used for chest pain in 523 patients admitted to the emergency department of Hospital Unimed Recife III}

\author{
Vitor Nunes de Miranda \\ Ricardo Pontes \\ Weidson Francisco Gonçalves Dantas \\ Izabel Albuquerque Libânia \\ Marcelo M. Valença \\ Fernando Cruz
}

Hospital da Unimed III, Unimed Recife, Recife, Pernambuco, Brasi

\section{$凶$}

Vitor Nunes de Miranda Unidade de Cardiologia, Hospital da Unimed III, Unimed Recife, Recife, Pernambuco, Brasil

Este artigo foi editado por Juliana Ramos de Andrade

Palavras-chave:

Dor torácica

Infarto do miocárdio

Fluxograma

Emergência

\section{Keywords:}

Chest pain

Myocardial infarction

Flowchart

Emergency

\section{Resumo}

Introdução A dor torácica é responsável por mais de 8 milhões de admissões por ano na emergência em hospitais nos EUA. Ainda não há um consenso sobre a melhor forma de se invesfigar ou tratar pacientes com dor torácica, além de ser uma área de constante aprimoramento nas diversas modalidades de como intervir naqueles pacientes onde o infarto do miocárdio é a causa da dor torácica. Recomenda-se o estabelecimento de um protocolo clínico e seu fluxograma no cuidado dos pacientes com dor torácica na emergência.

Objetivo Apresentam o fluxograma utilizado no Hospital da Unimed III, Unimed Recife, Recife, Brasil, para pacientes admitidos na Unidade de Emergência com d'or torácica. Com o objétivo de se avaliar dados relacionados com o uso do Protocolo Clínico de Dor Torácica de 2020, o número de pacientes que entraram no profocolo, quantos foram diagnosticados como infarto do miocárdio com supra ou sem supra, quantos pacientes conseguiram realizar o cateterismo em até 90 minutos e a letalidade. Uma revisão breve sobre dor torácica também foi realizada.

Método Dados sobre número de pacientes que entraram no protocolo de dor torácica foi obtido do prontuário eletrônico do paciente (PEP, Business Intelligence). O período estudado foi de $1^{\circ}$ de abril até 31 de dezembro de 2020 .

Resultados Foram 523 pacientes inseridos no protocolo. Dos 523 pacientes com dor torácica, 40 (7,7\%) receberam o diagnóstico de infarto do miocárdio, em 19/40 (47,5\%) com supra. Em $88 \%$ dos pacientes que foram submetidos a um cateterismo cardíaco o tempo porta-balão foi inferior a 90 minutos, mostrando a importância de um Protocolo Clínico na assistência de pacientes cardiológicos. Não houve morte entre os pacientes com infarto do miocárdio.

Conclusão Uma parcela significativa de pacientes com dor torácica por infarto do miocárdio recebeu intervençáo diagnostica e tratamento em intervalo de tempo considerado ideal para reperfusão do tecido miocárdico com isquemia. Não houve morte.

\section{Abstract}

Introduction Chest pain is responsible for more than 8 million hospital admissions per year in hospitals in the USA. There is still no consensus on the best way to investigate or reat patients with chest pain and be an area of constant improvement in the different modalities of how to intervene in those patients where myocardial infarction is the cause of chest pain. It is recommended to establish a clinical protocol and its flowchart in patients' care with chest pain in the emergency room.

Objective To present the flowchart used at the Hospital da Unimed III, Unimed Recife, Recife Brazil, for patients admitted to the Emergency Unit with chest pain. to evaluate the result of routine use of a clinical protocol, the number of patients who entered the protocol from Apri to December 2020 was verified. Also, the number of patients diagnosed with myocardial infarction with or without supra, how many of them were able to perform catheterization in up to 90 minutes, and lethality were determined. The authors carried out a brief review on chest pain.

Method Data on the number of patients who entered the chest pain protocol was obtained from the patient's electronic medical record (PEP, Business Intelligence). The period studied was from April 1 to December 31, 2020

Results There were 523 patients enrolled in the protocol. Of the 523 patients with chest pain $40(7.7 \%)$ were diagnosed with myocardial infarction, in $19 / 40(47.5 \%)$ with supra. In $88 \%$ of patients who underwent cardiac catheterization, the door-to-balloon time was less than 90 minutes, showing the importance of a Clinical Protocol in cardiac patients' care. There was no death among patients with myocardial infarction.

Conclusion A significant portion of patients with chest pain due to myocardial infarction received diagnostic intervention and treatment at an interval considered ideal for myocardial tissue reperfusion with ischemia. There was no death. 


\section{Introdução}

A dor torácica é responsável por mais de 8 milhões de admissões por ano na emergência em hospitais nos EUA. Ainda não há um consenso sobre a melhor forma de se investigar ou tratar pacientes com dor torácica, além de ser uma área de constante aprimoramento nas diversas modalidades de como intervir naqueles pacientes onde o infarto do miocárdio é a causa da dor torácica. ${ }^{1-10}$

O estabelecimento de um protocolo clínico no atendimento é essencial no caso de um paciente com dor torácica, principalmente por causa das formas atípicas de apresentação dos sintomas na isquemia do miocárdio, com formas muito variáveis de apresentação clínica entre um indivíduo e outro. ${ }^{6}$, 9, 11-14 Por exemplo, considerado o sexo do indivíduo, sabe-se que as mulheres com dor torácica têm, proporcionalmente, em relação aos homens, uma apresentação clínica de sintomatologia dolorosa de origem não isquêmica. ${ }^{15,} 160$ diagnóstico diferencial, portanto, com outras doenças que causam dor simulando um infarto do miocárdio é essencial no atendimento inicial do paciente, ${ }^{17}$ porque uma intervenção imediata e eficiente visando reperfusão do miocárdio é vital para preservação do tecido em isquemia. E não desejamos submeter indevidamente o paciente a procedimentos invasivos. Nesse contexto, em um estudo com pacientes sintomáticos, em mais de $50 \%$ dos deles a dor torácica não tinha como causa síndrome coronariana aguda. ${ }^{3}$

Frank e Lillian Gilbreth foram os primeiros a usarem um fluxograma como forma de demonstrar sequencialmente a forma planejada de uma atividade, quando em 1921 apresentaram "Gráficos do processo: primeiros passos para encontrar a melhor maneira de trabalhar" (Process Charts: First Steps in Finding the One Best Way to do Work) para membros da American Society of Mechanical Engineers (ASME). Desde então essa forma de planejamento tem sido utilizada amplamente, com normas estabelecidas nos anos 1960s pela American National Standards Institute (ANSI), que foram adotadas em 1970 pela The International Organization for Standardization (ISO) utilizando símbolos estabelecidos pela ANSI. O padrão atual, ISO 5807: 1985, foi revisado em $2019 .{ }^{18}$

Há uma diferença entre algoritmo e fluxograma: o algoritmo é um conjunto de critérios, ou instruções, estabelecidos sequencialmente para a execução de um determinado objetivo, ou solução, de um problema. $\bigcirc$ fluxograma é a representação gráfica do algoritmo.

Na Figura 1 é mostrado o fluxograma utilizado na Unimed Recife para pacientes que são admitidos com dor torácica. Desde o abril de 2020 o Protocolo Clínico para dor torácica foi incorporado no prontuário médico eletrônico no Hospital da Unimed III, obrigando a equipe médica e de enfermagem preencher o protocolo e estabelecer estratégia de atendimento ao paciente com dor torácica.

Com o objetivo de se avaliar dados relacionados com o uso do Protocolo Clínico de Dor Torácica usado no Hospital da Unimed Recife III, avaliamos o número de pacientes que entraram no protocolo, quantos foram diagnosticados como infarto do miocárdio com ou sem supra, quantos pacientes conseguiram realizar o cateterismo em até 90 minutos e a letalidade.

\section{Método}

Dados sobre número de pacientes que entraram no protocolo de dor torácica foi obtido do prontuário eletrônico do paciente (PEP, Business Intelligence). O período estudado foi de $1^{\circ}$ de abril até 31 de dezembro de 2020.

\section{Resultados}

No período de $1^{\circ}$ de abril até 31 de dezembro de 2020 foram atendidos na emergência do Hospital Unimed Recife III 523 pacientes com dor torácica. Na Tabela 1 encontramos dados relacionados com os 523 pacientes inseridos no protocolo. Dos 523 pacientes com dor torácica, 40 (7,7\%) receberam o diagnóstico de infarto do miocárdio. Em $88 \%$ dos pacientes que foram submetidos a um cateterismo cardíaco o tempo porta-balão foi inferior a 90 minutos, mostrando a importância de um Protocolo Clínico na assistência de pacientes cardiológicos.

Tabela 1. Dados relacionados com 523 pacientes com dor torácica que foram atendidos no Hospital Unimed Recife III e entraram no protocolo Clínico de Dor Torácica entre abril e dezembro de 2020

\begin{tabular}{|c|c|c|}
\hline Dados & Número & Percentagem \\
\hline IM com supra & $19 / 523$ & $3,6 \%$ \\
\hline IM sem supra & $21 / 523$ & $4,0 \%$ \\
\hline $\begin{array}{c}\text { Tempo porta-balão } \\
\text { até } 90 \text { minutos }\end{array}$ & $88 \%$ dos pacientes \\
\hline Letalidade & $0 \%$ \\
\hline IM, infarto do miocárdio & \\
\hline
\end{tabular}

\section{Discussão}

Um dos objetivos de um fluxograma para dor torácica é diminuir o desperdício em tempo entre o diagnóstico correto e reperfusão miocárdica, significando um melhor desfecho para o paciente, particularmente quando o tempo porta-balão é menor do que 90 minutos. Em um estudo realizado na Alemanha os intervalos entre (1) o primeiro contato médico até a admissão e (2) entre o início dos sintomas até a admissão do paciente foram, respectivamente, 58 minutos (35-118 $\mathrm{min}$ ) e 128 minutos (48-720 min); mostrando o problema do 


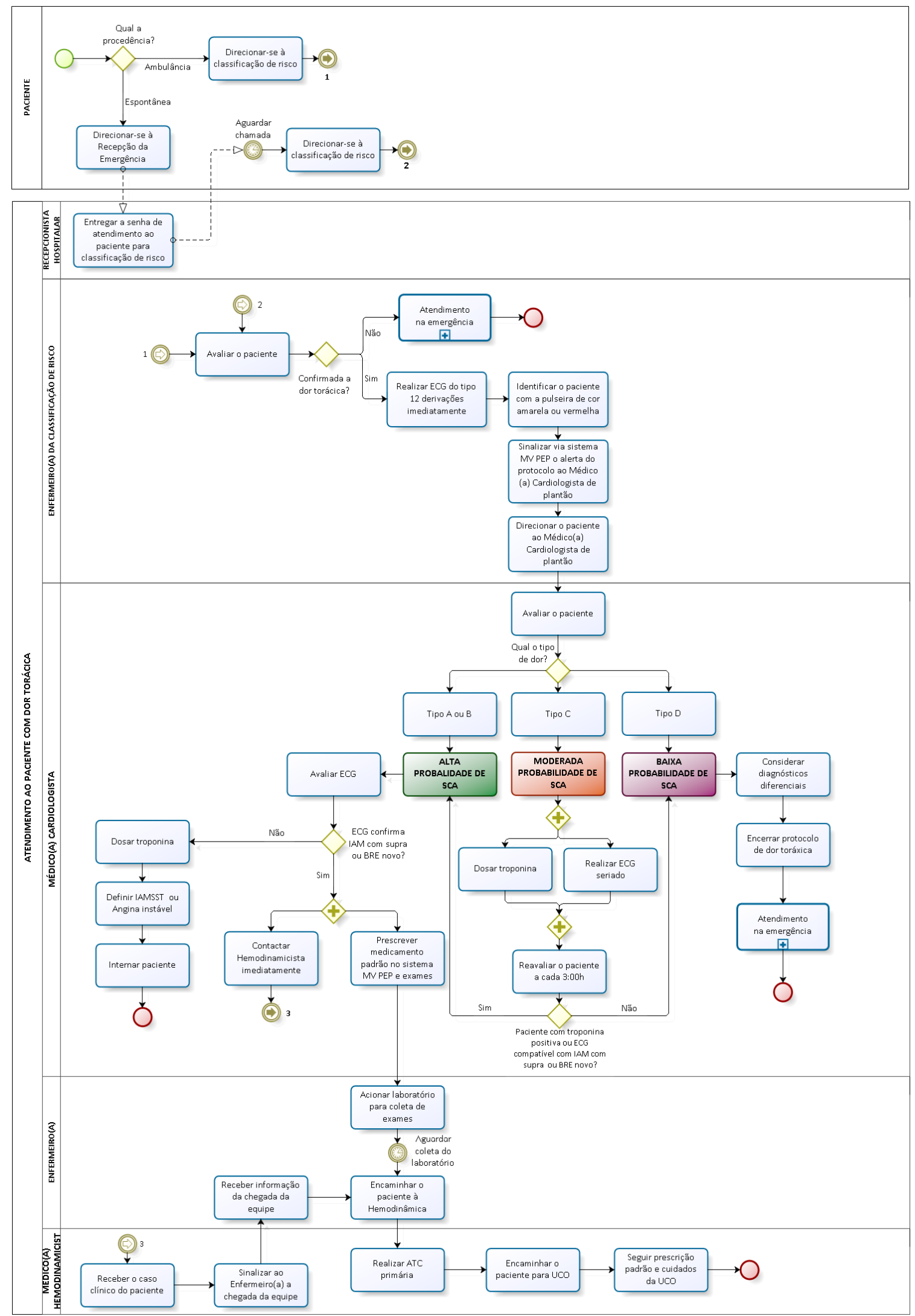

Figura 1. Fluxograma para dor torácica utilizado no Hospital Unimed Recife III. Obs.1: O tempo porta ECG é até 10 minutos, no qual o "tempo inicial" é o totem; Obs.2: Caso a hemodinâmica esteja indisponível, transferir o paciente para outro hospital (rede conveniada) até 2 horas da chegada do paciente ao Hospital Unimed Recife III; Obs.3: Caso não seja possível transferir o paciente, o cardiologista de plantão deverá fazer o trombolítico na emergência e encaminhar o paciente para Unidade Coronariana. 
atraso pré-clínico relacionado ao paciente até sua admissão na Unidade Coronariana. ${ }^{2,} 19,20$

Em outro estudo muito interessante analisou-se o intervalo de tempo até $o$ atendimento durante o horário normal de trabalho, comparando com o mesmo intervalo no atendimento fora do horário comercial; e o intervalo relacionado com o início dos sintomas até a admissão foi significativamente mais curto fora do expediente de trabalho. Também houve um número menor daqueles pacientes que esperam mais de 4 horas para serem atendido efetivamente $(33,0 \%$ vs. $43,1 \%, p<0,001) .^{2}$

Pacientes com elevação do segmento ST persistente foram submetidos a angiografia coronária na grande maioria dos casos, o que foi associado a uma alta taxa de intervenção coronária de aproximadamente 95\%.2, 19 Dados de 2012 revelaram um tempo médio da "porta à punção" de 31 minutos (1 1-75 min). Nesse estudo os autores descrevem que o tempo porta-agulha recomendado é $<60$ minutos. $^{2}$

\section{Conclusão}

Uma parcela significativa de pacientes com dor torácica por infarto do miocárdio recebeu intervenção diagnóstica e tratamento em intervalo de tempo considerado ideal para reperfusão do tecido miocárdico com isquemia. Não houve morte entre os pacientes que foram diagnosticados com infarto do miocárdio.

Concluímos que o uso do fluoxograma é essencial no atendimento de um paciente com dor torácica. Há também a necessidade de se ter disponível instalações hospitalares, com um serviço de hemodinâmica com equipamentos modernos, e equipe tecnicamente treinada para intervenções realizadas no menor intervalo de tempo possível, visando evitar um dano irreversível do miocárdio.

Conflito de interesses Os autores declaram não haver nenhum potencial conflito de interesses.

Contribuição dos autores $M M V$ e $F C$, Concepção e desenho da análise; IAL, coletou os dados; IAL, dados de contribuição ou ferramentas de análise; VNM, RP, MMV, escreveu o manuscrito; VNM, RP, WFGD, MMV, Revisão do conteúdo intelectual; VNM, RP, IAL, WFGD, MMV, FC Aprovação final do manuscrito concluído.

Victor Nunes de Miranda

https://orcid.org/

Ricardo Pontes

https://orcid.org/

Weidson Francisco Gonçalves Dantas

https://orcid.org/

Izabel Albuquerque Libânia

https://orcid.org/

Marcelo M. Valença

https://orcid.org/

Fernando Cruz

https://orcid.org/

\section{Referências}

1. Diarv T, Swain JM, Chang WT, et al. Early or First Aid Administration Versus Late or In-hospital Administration of Aspirin for Non-traumatic Adult Chest Pain: A Systematic Review. Cureus 2020; 12:e6862

2. Breuckmann F, Rassaf T, Hochadel M, et al. German chest pain unit registry: data review after the first decade of certification. Herz 2020

3. Vafaie M, Hochadel M, Munzel T, et al. Guideline-adherence regarding critical time intervals in the German Chest Pain Unit registry. Eur Heart J Acute Cardiovasc Care 2020;9:52-61

4. Arslan F, Voskuil M. The management of acute coronary syndromes in patients presenting without persistent ST-segment elevation: early invasive strategy for all? Neth Heart J 2017;25:170-172

5. Prejean SP, Din M, Reyes E, et al. Guidelines in review: Comparison of the 2014 AHA/ACC guideline for the management of patients with non-ST-elevation acute coronary syndromes and the 2015 ESC guidelines for the management of acute coronary syndromes in patients presenting without persistent ST-segment elevation. J Nucl Cardiol 2018;25:769-776

6. Harskamp RE, Laeven SC, Himmelreich JC, et al. Chest pain in general practice: a systematic review of prediction rules. BMJ Open 2019;9:e027081

7. Eggers KM, Jernberg $T$, Ljung $L$, et al. High-Sensitivity Cardiac Troponin-Based Strategies for the Assessment of Chest Pain Patients-A Review of Validation and Clinical Implementation Studies. Clin Chem 2018;64:1572-1585

8. Andruchow JE, Kavsak PA, McRae AD. Contemporary Emergency Department Management of Patients with Chest Pain: A Concise Review and Guide for the High-Sensitivity Troponin Era. Can J Cardiol 2018;34:98-108

9. Miley KL. Cardiac or Anxiety: A Literature Review of the Young Adult Patient Who Presents to the Emergency Department With Chest Pain. J Emerg Nurs 2016;42:108113

10. Roche T, Jennings N, Clifford S, et al. Review article: Diagnostic accuracy of risk stratification tools for patients with chest pain in the rural emergency department: A systematic review. Emerg Med Australas 2016;28:51 1-524

11. Boyle RSJ, Body R. The Diagnostic Accuracy of the Emergency Department Assessment of Chest Pain (EDACS) Score: A Systematic Review and Meta-analysis. Ann Emerg Med 2021;77:433-441

12. Mikhail M, Crowley G, Haider SH, et al. Non-Cardiac Chest Pain: A Review of Environmental Exposure-Associated Comorbidities and Biomarkers. EMJ Gastroenterol 2018;7:103-112

13. Lal A, Akhtar J, Isaac S, et al. Unusual cause of chest pain, Bornholm disease, a forgotten entity; case report and review of literature. Respir Med Case Rep 2018;25:270-273

14. Movahedian M, Afzal W, Shoja T, et al. Chest Pain Due to Pericardial Effusion as Initial Presenting Feature of Rheumatoid Arthritis: Case Report and Review of the Literature. Cardiol Res 2017;8:161-164

15. Settelmeier S, Rassaf T, Hochadel M, et al. Gender Differences in Patients Admitted to a Certified German Chest Pain Unit: Results from the German Chest Pain Unit Registry. Cardiology 2020; 145:562-569

16. Ziilstra LE, Bootsma M, Jukema JW, et al. Chest pain in the absence of obstructive coronary artery disease: A 
critical review of current concepts focusing on sex specificity, microcirculatory function, and clinical implications. Int J Cardiol 2019;280:19-28

17. Lo KB, Virk HUH, Lakhter V, et al. Clinical Outcomes After Treatment of Cocaine-Induced Chest Pain with Beta-Blockers: A Systematic Review and Meta-Analysis. Am J Med 2019;132:505-509

18. ISO 5807:1985 Information processing - Documentation symbols and conventions for data, program and system flowcharts, program network charts and system resources charts. 2019

19. Post F, Giannitsis E, Riemer T, et al. Pre- and early in-hospital procedures in patients with acute coronary syndromes: first results of the "German chest pain unit registry". Clin Res Cardiol 2012;101:983-991

20. Wechkunanukul K, Grantham H, Clark RA. Global review of delay time in seeking medical care for chest pain: An integrative literature review. Aust Crit Care 2017;30:13-20 